\title{
Cystic fibrosis newborn screening and detection of carriers
}

\section{Super}

\section{Detection of carriers can be managed}

A decision to introduce nationwide cystic fibrosis (CF) newborn screening in the United Kingdom has now been taken, although the exact procedure is still being determined, except in Scotland where screening began in February 2003. In Scotland, as well as in a number of regions in England and Wales where ad hoc newborn screening was started some time ago, an immune reactive trypsin (IRT)/ DNA system of newborn screening is used, DNA analysis being undertaken on those above a cut off point of IRT. The multiplex DNA arm covers about $86 \%$ of the most common mutations in the native UK population. ${ }^{1}$ There is evidence that the more severe mutations of the cystic fibrosis transmembrane regulator (CFTR) gene are the ones most likely to cause an increase in IRT. ${ }^{2}$

There are a number of advantages of IRT/DNA programmes: earlier full diagnosis within the first few weeks of life becomes possible for those in whom both mutations are found; there is a reduction in sweat testing and in the uncertainty that may follow inadequate sweat collection; also many fewer couples need to be faced with the worry that their baby might have CF than when IRT is used alone. ${ }^{3}$ In those from ethnic minorities where the mutation spectrum is largely unknown, one has no choice other than to proceed to sweat test in those with a persistently raised IRT.

The disadvantage of the IRT/DNA method is that the genetic analysis step results in the discovery of some healthy carrier infants, with the spectre of $\mathrm{CF}$ raised for the parents before the situation is resolved by negative sweat test. Two or three carrier newborns are discovered for each child diagnosed with CF. ${ }^{3}$

One issue that has concerned some is that one might discover non-paternity in the DNA arm of the test, when one tests the parents. Experience from our CF cascade programme ${ }^{5}$ is that, although we have discovered non-paternity of grandparents, none was found among the parents. This may indicate self selection in those parents who refuse tests. As long as no pressure is exerted on the parents to have themselves tested, those geneticists who participated in an email debate on this topic organised by Professor Rodney Pollitt in 2002 did not see discovery of non-paternity as a major problem.

Having discovered a carrier, what does one do with the information? There can be no argument that the parents must be informed of the result. For one thing, a sweat test is being organised on their infant to cover the possibility of $\mathrm{CF}$ with the second mutation not covered by the screening method; for another, both parents might be carriers with a risk of CF in future or existing children.

How much distress does the finding of a carrier newborn cause? Parsons and colleagues $^{6}$ sought to answer this specifically in a small questionnaire study based on newborn screening in Wales. Attitudes and feelings of parents were compared with those of a control group of mothers. The authors report their results with particular reference to the implications when the infant was found to be a carrier under the headings: attitude to newborn screening, infant carrier identification, adult carrier identification, the newborn screening protocol, and the family dimension. All carrier families were in favour of screening, and no evidence was found that the mother/baby relationship had been affected by carrier identification. None regarded their baby as ill, and most regarded the information that their baby was a carrier as useful and of relevance to the child in later life. Most also accepted the offer to have themselves tested. There were more initial negative feelings expressed by the parents found to be carriers about themselves than about their carrier infant: these included guilt at passing the gene on. However, by six months all had become philosophical about their carrier status. This accords with evidence that the CF carrier status is not associated with a diminished self concept or anxiety. ${ }^{7}$

In this Welsh study, the way the possibility of CF was first raised with the couple did cause anxiety, with a genetics nurse and health visitor calling together. It would appear that CF was explained in too great detail. One can see that this was inappropriate if one works out the chances that a neonate with a raised IRT and only one CF mutation will turn out to have CF. As about $12 \%$ of healthy newborn carriers of a CF gene have a raised IRT, ${ }^{4}$ then, from the known incidence of CF of 1 in 2500 and carrier incidence of 1 in 25, the odds would be 12 to 1 in favour of a newborn in whom only one mutation had been found being a carrier rather than a CF sufferer. Put another way, as, in about $75 \%$ of newborns with CF, both mutations would be identified using modern multiplex testing covering the common mutations in the native population of the United Kingdom and as there are two or three carriers identified for each affected individual, the odds of a single mutation in a neonate with a raised IRT indicating a carrier rather than affected would be somewhere between 8 and 12 to 1 or greater in parts of the country such as the northwest of England where a higher percentage of mutations are present.

In the study of Parsons et al there were two aspects of the screening protocol on which parents commented negatively. The one was refusal by the genetic counsellor (department) to check their other children to see if they were carriers. Parents saw an inconsistency in this. One of the authors (Angus Clarke) chaired a working party for the Clinical Genetics Society on genetic testing in children. ${ }^{8}$ The tone of this report was generally against such testing, suggesting that children should make their own decisions on whether to be tested when of an age to do so. It is refreshing to note that the authors are prepared to question their current practice in response to strongly expressed wishes of parents. The Genetic Interest Group (GIG) have long campaigned on behalf of parents in this respect, adopting the attitude expressed by many of their members, "Parents generally make decisions for the benefit of their families and children."

The other negative aspect that parents found most difficult was the suggestion that they contact their relatives to inform them of their potential carrier status. The authors suggest that their reluctance to do so signifies dysfunctional family relationships. I disagree with them on this and find the parents' behaviour understandable. They are in a state of grace after the birth of their healthy baby and are understandably

Abbreviations: CF, cystic fibrosis; IRT, immune reactive trypsin; CFTR, cystic fibrosis transmembrane regulator 
reluctant to cast a shadow over this. Their situation is entirely different from that of the parents of a child affected by the disease CF. Although active cascade testing of relatives may be an excellent idea in many circumstances, ${ }^{5}$ such practice applied to discovered carrier newborns is inappropriate. Simply mentioning that carrier testing for the extended family is available and giving the parents the pamphlet on cascade testing of relatives prepared by the Cystic Fibrosis Trust (available at http://www.cftrust.org.uk) should suffice, with no pressure on parents to contact relatives. The pamphlet contains all necessary information for those who wish to avail themselves of tests. In discussing the carrier state, while mentioning the very occasional disadvantage of some symptoms in common with $\mathrm{CF}$ in carriers, it also mentions the probable health advantage that the vast majority enjoy. This is likely to help people to see CF carrier status in a more positive light.

Following on from their experience, the authors suggest a very reasonable template of how to deal with results and the counselling issues. This includes extreme care in first making contact with parents when one CF mutation has been found. The primary health team known to the parents should be asked to convey the information in a positive light, with the genetics team not involved at this stage and with details of the disease kept to a minimum. Printed advice to primary care teams on how to deal with these issues should be prepared by the National Newborn Screening Programme Centre and distributed by the Department of Health.

In a larger study than that reported by Parsons et al, Wheeler and colleagues ${ }^{9}$ discuss their experiences with the parents of 102 newborns discovered to have one CF mutation in an IRT/DNA screening programme in Massachusetts. The parents were counselled in the genetics department on the same day as the sweat test, in the hour before the result became known. As there was a low chance that the child would have CF, discussion of the disease was brief and more for the purposes of explaining why carrier testing was being offered to the parents. In their study, most parents gave the chance that they might both be carriers as their reason for opting to have themselves tested. Although it would require some organisation, this is an approach in which clinical genetics and IRT/DNA screening units could fruitfully cooperate in the United Kingdom. Again it would be useful to have written national protocols to follow.

The advantages of including DNA analysis in newborn CF screening outweigh the disadvantages, but special care must be taken in managing the parents of neonates discovered to be carriers.

Arch Dis Child Fetal Neonatal Ed 2003;88:F448-F449

\section{Author's affiliation}

M Super, Royal Manchester Children's Hospital, Manchester M27 4HA, UK

Correspondence to: Dr Super;

maurice.super@man.ac.uk

\section{REFERENCES}

1 Schwarz MJ, Malone G, Haworth A, et al. Cystic fibrosis mutation analysis: report from $22 \mathrm{UK}$ Regional Genetics Laboratories. Hum Mutation 1995;6:326-333.

2 Lecoq I, Brouard J, Ferec C, et al. Blood immunoreactive trypsin concentrations are genetically determined in healthy and cystic fibrosis newborns. Acta Paediatr 1999;88:338-41.

3 Ranieri E, Lewis BD, Gerace RL, et al. Neonatal screening for cystic fibrosis using immunoreactive trypsin and direct gene analysis: four years' experience. $B M$ 1994;308: 1469-72.

4 Scotet V, de Braekeleer M, Audrezet M-P, et al. Prevalence of CFTR mutations in hypertrypsinaemia detected through neonatal screening of cystic fibrosis. Clin Genet 2001;59:42-7.

5 Super M, Schwarz MJ, Malone G, et al. Active cascade testing for cystic fibrosis carriers. BMJ 1994;308:1462-67.

6 Parsons EP, Clarke AJ, Bradley DM. Implications for carrier identification in newborn screening for cystic fibrosis. Arch Dis Child 2003;88.

7 Denayer L, Welkenhuyzen M, Evers-Kiebooms G, et al. The CF carrier status is not associated with diminished self-concept or increased anxiety: results of psychometric testing after at least 1 year. Clin Genet 1996;49:232-6.

8 Clinical Genetics Society (UK) The genetic testing of children. Report of a working party of the Clinical Genetics Society. J Med Genet 1994;72:785-97.

9 Wheeler PG, Smith R, Dorkin H, et al. Genetic counselling after implementation of state-wide cystic fibrosis newborn screening: two years' experience in one medical centre. Genet Med 2001;3:411-15. 